\title{
The Influence of Media Treatments on Color Changes, Dimensional Stability, and Cracking Behavior of Bamboo Scrimber
}

\author{
Jingpeng Li $\mathbb{D}^{1,2}$ Yuhe Chen, ${ }^{1,2}$ Jun Xu, ${ }^{1}$ Danjing Ren, ${ }^{1}$ Hui Yu, ${ }^{1,2}$ \\ Fan Guo, 1 and Zaixing $W u$ iD ${ }^{1,2}$ \\ ${ }^{1}$ Key Laboratory of High Efficient Processing of Bamboo of Zhejiang Province, China National Bamboo Research Center, \\ Hangzhou 310012, China \\ ${ }^{2}$ Engineering Technology Research Center for Building and Decorating Materials of Bamboo State Forestry Administration, \\ Hangzhou 310012, China \\ Correspondence should be addressed to Jingpeng Li; lijp@caf.ac.cn and Zaixing Wu; jansonwu@126.com
}

Received 9 October 2017; Revised 25 December 2017; Accepted 25 February 2018; Published 28 March 2018

Academic Editor: Chaoqun Zhang

Copyright (c) 2018 Jingpeng Li et al. This is an open access article distributed under the Creative Commons Attribution License, which permits unrestricted use, distribution, and reproduction in any medium, provided the original work is properly cited.

\begin{abstract}
The purpose of this study is to evaluate the influence of media treatments on color changes, dimensional stability, and cracking behavior of bamboo scrimber, which has many applications in construction. The bamboo scrimber specimens comprised bamboo bundles with a low molecular weight phenol formaldehyde resin which were manufactured via a cold-in and cold-out hot-pressing process. Small clear specimens were sampled and treated under ten different conditions: water immersion at $25^{\circ} \mathrm{C}, 63^{\circ} \mathrm{C}$, and $100^{\circ} \mathrm{C}$; oil immersion at $100^{\circ} \mathrm{C}$ and $150^{\circ} \mathrm{C}$; air drying at $63^{\circ} \mathrm{C}, 100^{\circ} \mathrm{C}$, and $150^{\circ} \mathrm{C}$; and infrared drying at $100^{\circ} \mathrm{C}$ and $150^{\circ} \mathrm{C}$. Then, tests were conducted to determine the color changes, dimensional stability, and cracking behavior of the bamboo scrimbers. The results showed that the bamboo scrimber specimens became darker after all treatment conditions, especially the oil treatments and infrared drying. The color of the oil-treated bamboo scrimbers was found to be more homogenous than the others. The dimensional stability of the bamboo scrimbers was more or less influenced by the water treatments, air drying, and infrared drying, and the oil-treated bamboo scrimbers were relatively stable. Moreover, during the 4-hour treatments, cracks were found in the bamboo scrimbers after air drying at $150^{\circ} \mathrm{C}$ or infrared drying at $100^{\circ} \mathrm{C}$ or $150^{\circ} \mathrm{C}$.
\end{abstract}

\section{Introduction}

Bamboo scrimbers are increasingly explored as a material with significant potential for construction applications [1]. Bamboo scrimbers could be the ideal materials to fulfil the demand of wood in the construction sector because bamboo grows rapidly and matures to structural strength within 3-5 years [2]. Bamboo is a sustainable resource, abundantly available, and biodegradable, and it is widely used in producing bamboo-based composites such as bamboo scrimber, laminated bamboo lumber, bamboo plywood, and bamboo particle board $[3,4]$. Compared with other bamboo-based composites, bamboo scrimbers have received an increasing amount of attention due to their higher utilization ratio and excellent physical and mechanical properties, which have extensive applications in flooring, furniture, buildings, and the civil engineering field.

Recently, a significant amount of research has been devoted to study the physical and mechanical properties of bamboo scrimbers. Sharma et al. [5] studied the mechanical properties of two types of commercially available products-bamboo scrimber and laminated bamboo sheets-and compared these to that of timber and engineered timber products. They found that both bamboo products exhibited comparable mechanical properties to other structural materials such as timber and raw bamboo. Sharma et al. [6] also evaluated the effect of processing methods on the mechanical properties of engineered bamboo. Kumar et al. [7] investigated the influence of bamboo scrimber densities on the mechanical and water absorption properties. They 


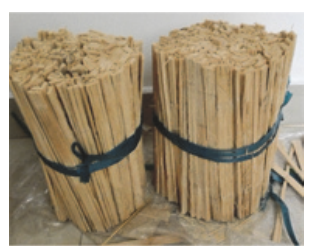

Bamboo bundles

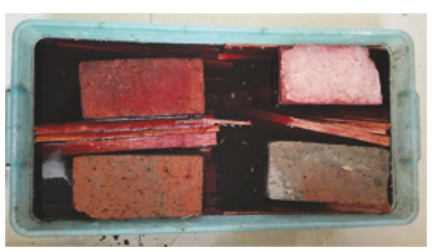

Impregnating

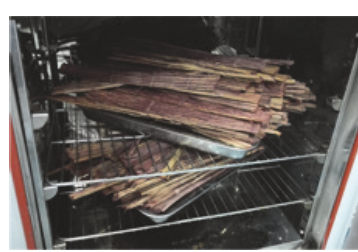

Drying

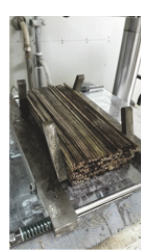

Assembling

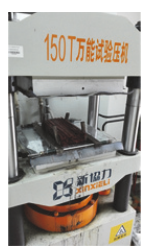

Hot pressing Bamboo scrimber

FIgURE 1: General manufacturing process of bamboo scrimbers in the laboratory.

concluded that the density had a significant influence on the mechanical and water absorption properties of bamboo scrimber. Yu et al. [8] investigated the use of bamboo as a promising raw material for fabricating bamboo scrimbers. Their study aimed to determine the water absorption, dimensional stability, and mechanical properties of bamboo scrimbers with different weight percentages of phenol formaldehyde resin and different heat treatments of bamboo bundles. They also investigated the mechanical properties of bamboo fiber-reinforced composite and compared these to that of commercial bamboo scrimber and laminated bamboo lumber. They presented the tensile, compressive, and shear strengths of a bamboo scrimber with a density of $1100 \mathrm{~kg} / \mathrm{m}^{3}$ [9]. Zhu et al. [10] evaluated the physical and mechanical properties of bamboo scrimbers subjected to outdoor exposure. They studied the surface color, modulus of rupture, modulus of elasticity, and swelling properties of bamboo scrimbers after 2 years of exposure. Xie et al. [11] evaluated the influence of fabricated density and bamboo species on the physical-mechanical properties of bamboo fiber bundlereinforced composites (BFCs). They found that BFCs with a density of $1200 \mathrm{~kg} / \mathrm{m}^{3}$ exhibited lower water absorption, better dimensional stability, and higher mechanical properties compared to those with a lower density. Shuangguan et al. [12] studied the physical characteristic, mechanical properties, and chemical composition of heat-treated bamboo scrimber. Du et al. [13] investigated the influence of different application methods of a fire retardant, that is, $\left(\mathrm{NH}_{4}\right)_{2} \mathrm{HPO}_{4}$, on the physical and mechanical properties of bamboo scrimber.

However, there is little research regarding the effect of media treatments on the changes of bamboo scrimber itself, either domestic or overseas, especially the dimensional stability and color changes. The present study investigates the influences of media treatments, such as water immersion, oil immersion, air drying, and infrared drying, on color changes, dimensional stability, and cracking behavior of bamboo scrimbers.

\section{Materials and Methods}

2.1. Materials. Moso bamboo bundles (Phyllostachys pubescens) with an age of 3-4 years were purchased from Anji, Zhejiang province. The maximum diameter of bundles was between $3.11 \mathrm{~mm}$ and $4.82 \mathrm{~mm}$ with moisture content of approximately $10 \%$. The matrix material used in this study was based on a commercially available low molecular weight phenol formaldehyde resin, with the following parameters:
TABLE 1: Media treatments of bamboo scrimbers.

\begin{tabular}{lc}
\hline Name & Treatment conditions \\
\hline A & Water immersion at $63^{\circ} \mathrm{C}$ \\
B & Water immersion at $100^{\circ} \mathrm{C}$ \\
C & Hot oil immersion at $100^{\circ} \mathrm{C}$ \\
D & Hot oil immersion at $150^{\circ} \mathrm{C}$ \\
E & Air drying at $63^{\circ} \mathrm{C}$ \\
F & Air drying at $100^{\circ} \mathrm{C}$ \\
G & Air drying at $150^{\circ} \mathrm{C}$ \\
$\mathrm{H}$ & Infrared drying at $100^{\circ} \mathrm{C}$ \\
J & Infrared drying at $150^{\circ} \mathrm{C}$ \\
K & Water immersion at $25^{\circ} \mathrm{C}$ \\
\hline
\end{tabular}

solid content of $45 \%$, viscosity of $38 \mathrm{cP} \cdot \mathrm{s}$, and $\mathrm{pH}$ value of 12. It was supplied by Nanjing Dynea Chemical Industry Co., Ltd.

2.2. Preparation of Bamboo Scrimbers. The bamboo scrimbers comprised bamboo bundles with a low molecular weight phenol formaldehyde resin which were manufactured via a cold-in and cold-out hot-pressing process [8]. The process of manufacturing bamboo scrimber is shown in Figure 1. The resin-impregnated bamboo bundles were assembled along the grain direction in the hot-pressing mold. When the temperature of the hot plate heated up to $55^{\circ} \mathrm{C}$, the bamboo mat was put into the press. The pressure was kept at $5.5 \mathrm{MPa}$ according to relative density at $140 \pm 2^{\circ} \mathrm{C}$ for $30 \mathrm{~min}$ to cure the mat. Afterwards, cold water was introduced into the hot plate and cooled down to $55^{\circ} \mathrm{C}$. The pressure was released and the cured mat was removed from the press. The nominal dimension of bamboo scrimber was $300 \times 180 \times 13 \mathrm{~mm}^{3}$. The resulting products were tested as a final product with no additional modifications. The average density of the bamboo scrimber specimens was $1080 \mathrm{~kg} \cdot \mathrm{m}^{-3}$. All specimens were conditioned in a room under a controlled environment at $20 \pm 2^{\circ} \mathrm{C}$ and $65 \pm 3 \%$ relative humidity (RH) for 2 weeks prior to testing.

2.3. Media Treatments of Bamboo Scrimbers. Media treatments were carried out under ten different conditions as shown in Table 1 . The duration of all treatments were 4 hours. During the tests, the specimens, with dimensions of $40 \times 20$ $\times 13 \mathrm{~mm}^{3}$ (length $\times$ width $\times$ thickness), were measured every 30 minutes. Fifteen replicate tests were conducted at each treatment condition. 
TABLE 2: Effect of media treatments on the color change of bamboo scrimbers.

\begin{tabular}{|c|c|c|c|c|c|c|c|c|c|c|}
\hline \multirow{2}{*}{ Treatment } & \multicolumn{3}{|c|}{ Color in CIE $L^{*} a^{*} b^{*}$ system } & \multicolumn{4}{|c|}{ Color difference } & \multicolumn{3}{|c|}{ Change (\%) } \\
\hline & $L^{*}$ & $a^{*}$ & $b^{*}$ & $\Delta L^{*}$ & $\Delta a^{*}$ & $\Delta b^{*}$ & $\Delta E^{*}$ & $\Delta L^{*}$ & $\Delta a^{*}$ & $\Delta b^{*}$ \\
\hline Untreated & 56.9 & 10.5 & 22.5 & -- & -- & -- & -- & -- & -- & -- \\
\hline A & 56.2 & 10.4 & 21.9 & -0.7 & -0.1 & -0.6 & 0.9 & -1.23 & -0.95 & -2.67 \\
\hline B & 54.6 & 10.7 & 23.8 & -2.3 & 0.2 & 1.3 & 2.6 & -4.04 & 1.90 & 5.78 \\
\hline $\mathrm{C}$ & 44.4 & 12.2 & 19.9 & -12.5 & 1.7 & -2.6 & 12.9 & -22.24 & 16.35 & -11.87 \\
\hline $\mathrm{D}$ & 39.9 & 10.9 & 16.6 & -17.0 & 0.4 & -5.9 & 18.0 & -31.14 & 3.74 & -24.79 \\
\hline E & 56.0 & 10.9 & 22.3 & -0.9 & 0.4 & -0.2 & 1.0 & -2.03 & 3.28 & -1.01 \\
\hline $\mathrm{F}$ & 56.8 & 11.2 & 23.1 & -0.1 & 0.7 & 0.6 & 0.9 & -0.25 & 6.42 & 3.61 \\
\hline G & 49.4 & 10.8 & 19.5 & -7.5 & 0.3 & -3.0 & 8.1 & -13.39 & 2.75 & -13.45 \\
\hline $\mathrm{H}$ & 49.5 & 11.1 & 18.6 & -7.4 & 0.6 & -3.9 & 8.4 & -13.03 & 5.36 & -16.88 \\
\hline J & 29.9 & 6.3 & 7.2 & -27.0 & -4.2 & -15.3 & 31.3 & -54.66 & -38.89 & -78.46 \\
\hline K & 53.3 & 11.4 & 23.5 & -3.6 & 0.9 & 1.0 & 3.8 & -7.27 & 8.11 & 5.38 \\
\hline
\end{tabular}

2.4. Color Measurements. The surface color of the bamboo scrimber specimens was measured with a color measurement instrument according to the CIELAB color system (instrument: Konica Minolta Color Reader (Minolta CR-10, Osaka, Japan); diameter of measured circle: $10 \mathrm{~mm}$; light source: Standard Illuminant D65, which represents normal daylight including the UV region; and 108 standard observers), where $L^{*}$ is the measure of lightness, $a^{*}$ is the chroma from green to red, and $b^{*}$ is the chroma from blue to yellow. The color difference is defined by the following equation:

$$
\Delta E^{*}=\sqrt{\left(\Delta L^{*}\right)^{2}+\left(\Delta a^{*}\right)^{2}+\left(\Delta b^{*}\right)^{2}}
$$

where $\Delta E^{*}$ is the color difference, $\Delta L^{*}$ is the lightness difference, and $\Delta a^{*}$ and $\Delta b^{*}$ are the chroma differences. $\Delta$ means the differences between the initial and final parameters of the samples after the treatments. $L^{*}, a^{*}$, and $b^{*}$ are the average values of six locations on each sample.

2.5. Evaluation of Physical Properties. The weight percentage change of the samples was based on the weight of the specimens before and after the $4 \mathrm{~h}$ treatments (measured every 30 minutes). For the water immersions and oil treatments, the weights of the treated samples were measured immediately after the removal of the surface media with a dry cotton fabric. The same specimens were used for the determination of dimensional stability. The properties were evaluated based on thickness change and width change after the treatments. One hundred and twenty specimens were prepared, and the changes in the width and thickness of all samples were measured before and after the treatments. The weight change and dimensional stability (width change and thickness change) of the specimens were calculated from the following equations.

$$
\text { Weight percentage change }(\%)=\frac{M_{1}-M_{0}}{M_{0}} \times 100 \text {, }
$$

where $M_{0}$ is the weight $(\mathrm{g})$ before treatment and $M_{1}$ is the weight $(\mathrm{g})$ after treatment.

$$
\text { Width percentage change }(\%)=\frac{W_{1}-W_{0}}{W_{0}} \times 100 \text {, }
$$

where $W_{0}$ is the width (mm) before treatment and $W_{1}$ is the width $(\mathrm{mm})$ after treatment.

$$
\text { Thickness percentage change }(\%)=\frac{T_{1}-T_{0}}{T_{0}} \times 100 \text {, }
$$

where $T_{0}$ is the thickness $(\mathrm{mm})$ before treatment and $T_{1}$ is the thickness $(\mathrm{mm})$ after treatment.

2.6. Evaluation of Cracking Behavior of Bamboo Scrimbers. The cracking behaviors of the bamboo scrimbers were analyzed by a stereomicroscope (Olympus SZ61, Tokyo, Japan) attached to a computer with an appropriate magnification to observe the cross-section of the bamboo scrimbers after the 4-hour media treatments.

\section{Results and Discussion}

3.1. The Effect of Media Treatments on the Color Changes of Bamboo Scrimbers. The results of changes in color $\left(\Delta E^{*}\right)$ and lightness $\left(\Delta L^{*}\right)$ of bamboo scrimbers after the 4-hour media treatments are presented in Table 2. The $L^{*}$ values of the bamboo scrimbers are shown in Table 2. The decrease in $L^{*}$ for all treatments indicates that the specimens became darker after the 4-hour treatments, especially the treatments of $C, D, G, H$, and J. The values were -12.5 for $C,-17.0$ for $D,-7.5$ for $G,-7.4$ for $H$, and -27.0 for J. Darkening as a result of the media treatments was also clearly visible in Figure 2, and we found that it increased with treatment time. Similar results were observed by other researchers [14-16]. The change in the color of the bamboo scrimbers during the treatments may be due to the degradation of hemicellulose and other noncellulosic polysaccharides [14, 17-19]. As shown in Table 2, the lightness of the bamboo scrimbers decreased $1.23 \%$ (A), 4.04\% (B), 22.24\% (C), 31.14\% (D), 2.03\% (E), 


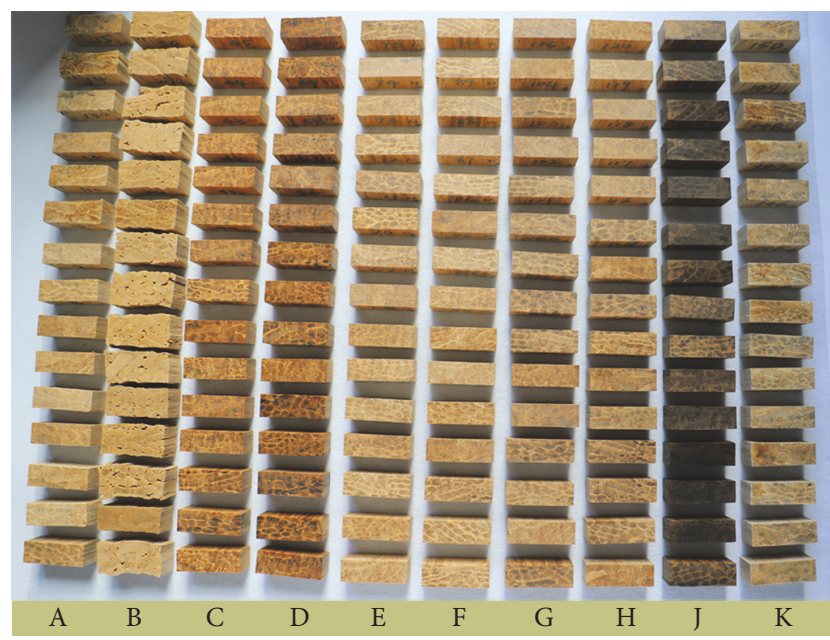

FIGURE 2: Digital photograph of bamboo scrimbers after media treatments.

0.25\% (F), $13.39 \%(\mathrm{G}), 13.03 \%(\mathrm{H}), 54.66 \%(\mathrm{~J})$, and $7.27 \%$ (K). The maximum reduction was obtained for the treatment of infrared drying at $150^{\circ} \mathrm{C}$ for $4 \mathrm{~h}$. As Figure 2(J) shows, the surface color of the bamboo scrimber turned dark after 4 hours of infrared drying. The green-red $\left(a^{*}\right)$ and yellowblue $\left(b^{*}\right)$ color coordinates also changed significantly under the treatment conditions. The positive values of $\Delta a^{*}$ indicate a tendency of the sample surface to become reddish. The media-treated bamboo scrimbers showed a more reddish color except for the treatments of A and J, which showed a more greenish color. It is interesting note that the $\Delta a^{*}$ value of treatment $\mathrm{C}$ was higher than that of treatment $\mathrm{D}$. The color of bamboo scrimber may be affected by oil uptake [20]. The $b^{*}$ values of samples are also presented in Table 2. The positive values of $\Delta b^{*}$ indicate a tendency of the specimen surface to become yellow, while the negative values mean a tendency to become blue. For B, F, and K, after the 4hour treatments, these specimens became yellower, while the others became bluer. The maximum reduction of $\Delta b^{*}$ was obtained for the treatment of infrared drying at $150^{\circ} \mathrm{C}$ for $4 \mathrm{~h}$. The greater value in the changes of $\Delta b^{*}$ compared to $\Delta a^{*}$ indicates that yellowing of the treated bamboo scrimbers is more dominant than the reddish changes. The total color changes $\left(\Delta E^{*}\right)$ of the treated bamboo scrimbers are given in Table 2. Our results showed that $\Delta E^{*}$ remarkably increased after the oil and infrared drying treatments. These results are in accordance with the results of Figure 2.

3.2. The Effect of Media Treatments on the Weight Percentage Changes of Bamboo Scrimbers. Figure 3 shows the effect of media treatments on the mean weight percentage change of the treated specimens. As shown in Figure 3, the weights of bamboo scrimbers increased after treatments of A, B, $\mathrm{C}$, and $\mathrm{K}$. The gain in the specimen weight increased with the increase of treatment time. As Figure 3 (A, B, and K) shows, the water absorption of bamboo scrimbers increased with the treatment temperature. For the $4 \mathrm{~h}$ treatments, the weight percentage change values of bamboo scrimbers after treatments of A, B, and K were approximately $13.57 \%, 35.51 \%$,

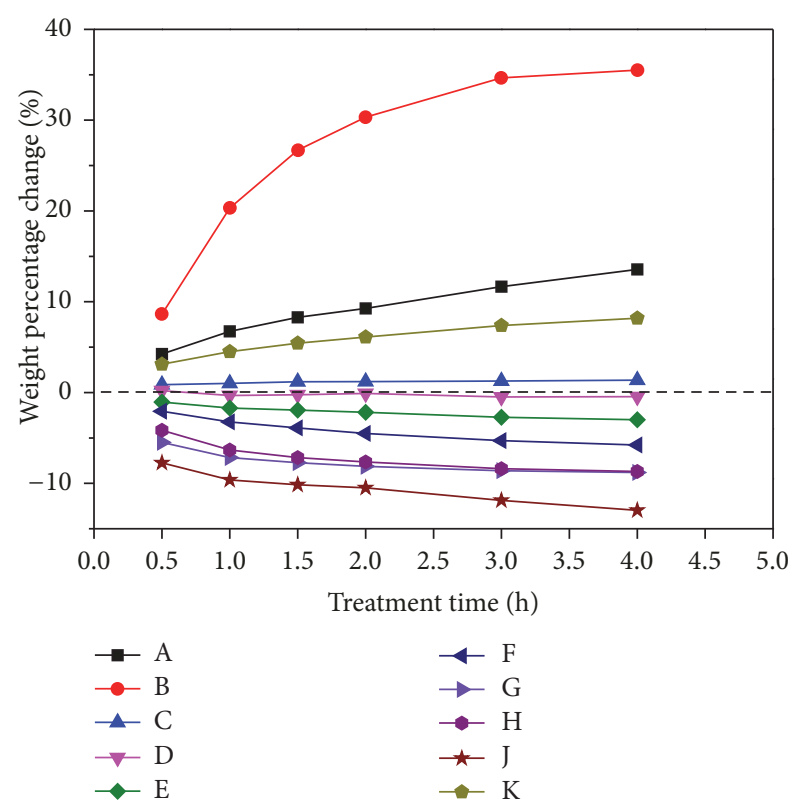

FIGURE 3: Weight percentage change of bamboo scrimbers in different media at different treatment temperatures. $\mathrm{A}, \mathrm{B}$, and $\mathrm{K}$ : water immersion at $63^{\circ} \mathrm{C}, 100^{\circ} \mathrm{C}$, and $25^{\circ} \mathrm{C}$; $\mathrm{C}$ and $\mathrm{D}$ : oil immersion at $100^{\circ} \mathrm{C}$ and $150^{\circ} \mathrm{C} ; \mathrm{E}, \mathrm{F}$, and $\mathrm{G}$ : air drying at $63^{\circ} \mathrm{C}, 100^{\circ} \mathrm{C}$, and $150^{\circ} \mathrm{C}$; $\mathrm{H}$ and $\mathrm{J}$ : infrared drying at $100^{\circ} \mathrm{C}$ and $150^{\circ} \mathrm{C}$.

and $8.19 \%$, respectively. Compared with the water treatment, the oil treatment did not lead to an obvious change in the weight of bamboo scrimbers. The weight percentage change value was only $1.37 \%$. When the oil temperature increased to $150^{\circ} \mathrm{C}$, during the 4-hour treatments, the weights of bamboo scrimbers decreased with the treatment time (Figure 3(D)). The weight percentage change value was $-0.45 \%$. Compared to treatment $\mathrm{D}$, the weights of bamboo scrimbers remarkably decreased during the treatment time. In particular, for the treatment of J, the weights of bamboo scrimbers obviously decreased after $2 \mathrm{~h}$ of treatment. The weight percentage change values of bamboo scrimbers after treatments of $\mathrm{E}, \mathrm{F}$, $\mathrm{G}, \mathrm{H}$, and J were approximately $-2.99 \%,-5.78 \%,-8.80 \%$, $-8.69 \%$, and $-12.97 \%$, respectively. For both air drying and infrared drying, the weight percentage change values of bamboo scrimbers increased with the treatment temperature. As the treatment temperature increases, most of the thermally weak polymeric components, that is, hemicelluloses, begin to degrade, resulting in the production of methanol, acetic acid, and various volatile heterocyclic compounds [14, 16]. Undeniably, the water loss in bamboo scrimbers was the primary cause for the decrease in the weight of the specimens.

3.3. The Effect of Media Treatments on Dimensional Stability of Bamboo Scrimbers. Figures 4 and 5 present the results of width percentage change and thickness percentage change of the differently treated bamboo scrimbers, respectively. As the figures show, the water immersions can sensitively change the dimension of bamboo scrimbers, especially the treatment of $\mathrm{B}$. The width percentage change and thickness percentage 


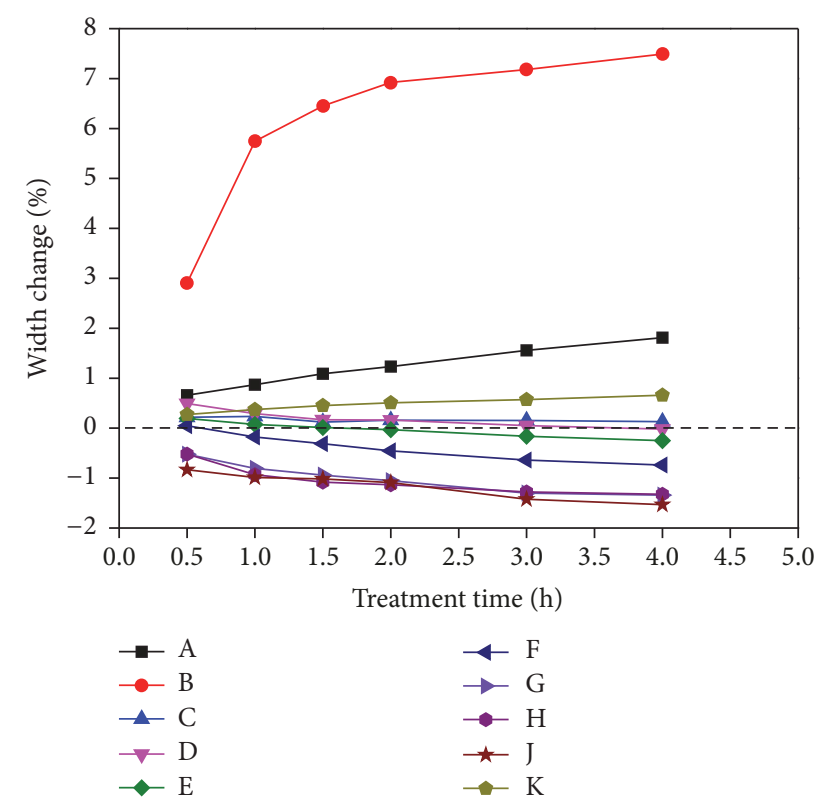

FIGURE 4: Width percentage change of bamboo scrimbers during 4hour treatments. $\mathrm{A}, \mathrm{B}$, and $\mathrm{K}$ : water immersion at $63^{\circ} \mathrm{C}, 100^{\circ} \mathrm{C}$, and $25^{\circ} \mathrm{C}$; C and D: oil immersion at $100^{\circ} \mathrm{C}$ and $150^{\circ} \mathrm{C}$; E, F, and G: air drying at $63^{\circ} \mathrm{C}, 100^{\circ} \mathrm{C}$, and $150^{\circ} \mathrm{C} ; \mathrm{H}$ and J: infrared drying at $100^{\circ} \mathrm{C}$ and $150^{\circ} \mathrm{C}$.

change of the treated bamboo scrimbers after 4-hour treatments of $\mathrm{A}, \mathrm{B}$, and $\mathrm{K}$ increased with the temperature. The width percentage change values after 4 -hour treatments of $\mathrm{A}, \mathrm{B}$, and $\mathrm{K}$ were $1.81 \%, 7.49 \%$, and $0.66 \%$, and the thickness percentage change values were $12.13 \%, 58.71 \%$, and $2.14 \%$, respectively. Compared with the water treatment, the oil treatment did not obviously change the dimension of the bamboo scrimbers. After 4-hour treatments of $\mathrm{C}$ and $\mathrm{D}$, the width percentage change values of the bamboo scrimbers were only $0.129 \%$ and $-0.018 \%$, respectively. In the thickness change study, after 4 -hour treatments, the $100^{\circ} \mathrm{C}$ oil-treated bamboo scrimbers showed less swelling than the $150^{\circ} \mathrm{C}$ oiltreated samples. The thickness percentage change value was only $0.541 \%$ for treatment C, but $4.095 \%$ for treatment D. For all of the air drying and infrared drying treatments, as shown in Figure 4 (E, F, G, H, and J), the width percentage change values of the bamboo scrimbers decreased with the treatment time. For the $4 \mathrm{~h}$ treatments, the width percentage change values after treatments of E, F, G, H, and J were approximately $-0.25 \%,-0.74 \%,-1.34 \%,-1.32 \%$, and $-1.53 \%$, respectively. The bamboo scrimbers after 4 hours of infrared drying at $150^{\circ} \mathrm{C}$ showed the greatest width shrinkage of $1.53 \%$. However, the thickness of the bamboo scrimbers increased $2.20 \%$ after 4 hours of infrared drying at $150^{\circ} \mathrm{C}$ as shown in Figure 5 inset. This could be explained by the gradual decrease of water in the bamboo scrimbers during the 4-hour infrared drying. The rate of water loss in and out of the bamboo scrimbers was not uniform. This can quickly lead to the cracking of the bamboo scrimbers after 0.5 hours of infrared drying, which caused an increase in the thickness. The results were supported by the following cracking research.

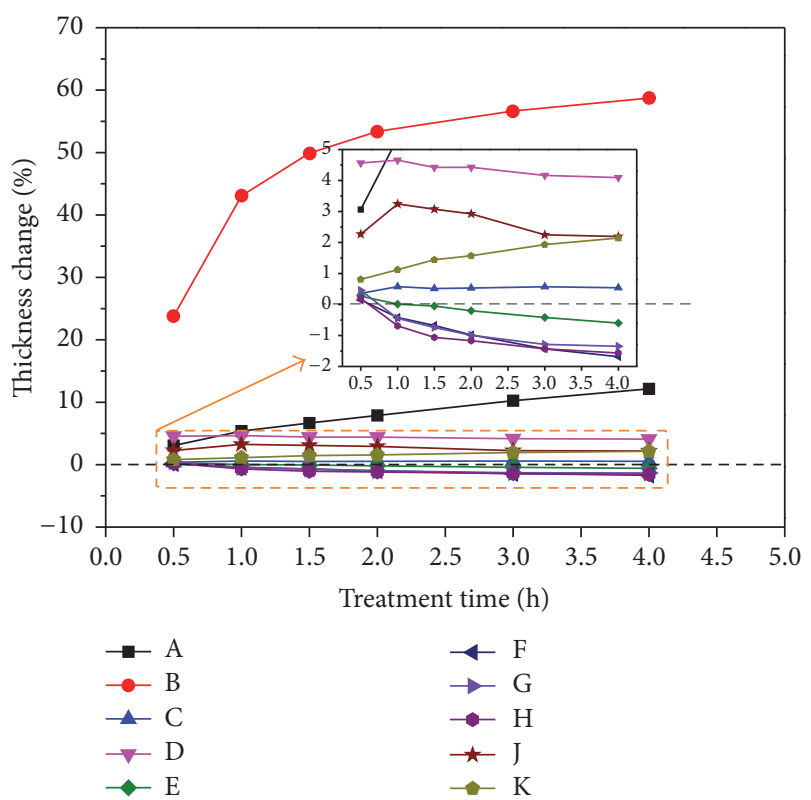

FIGURE 5: Thickness percentage change of bamboo scrimbers during 4-hour treatments. $\mathrm{A}, \mathrm{B}$, and $\mathrm{K}$ : water immersion at $63^{\circ} \mathrm{C}, 100^{\circ} \mathrm{C}$, and $25^{\circ} \mathrm{C}$; $\mathrm{C}$ and $\mathrm{D}$ : oil immersion at $100^{\circ} \mathrm{C}$ and $150^{\circ} \mathrm{C}$; E, F, and G: air drying at $63^{\circ} \mathrm{C}, 100^{\circ} \mathrm{C}$, and $150^{\circ} \mathrm{C} ; \mathrm{H}$ and J: infrared drying at $100^{\circ} \mathrm{C}$ and $150^{\circ} \mathrm{C}$.

3.4. The Effect of Media Treatments on Cracking Behavior of Bamboo Scrimbers. The cracking behavior is one of the most important properties of bamboo scrimbers and is used to evaluate the quality of bamboo products. The effects of media treatments on the cracking behavior of bamboo scrimbers during the 4-hour treatments are presented in Figures 6 and 7. As Figure 6 shows, after 4-hour immersion in water at $25^{\circ} \mathrm{C}(\mathrm{K})$ and $63^{\circ} \mathrm{C}(\mathrm{A})$, no surface cracks were observed in the cross-section of treated bamboo scrimbers. However, when the water temperature was raised to $100^{\circ} \mathrm{C}$ (Figure 6(B)), some cracks appeared in the bamboo scrimber after only 0.5 hours of treatment. The size and number of cracks increased with the treatment time. Water absorption affected the physical properties of bamboo scrimbers, as well as the matrix structure and fiber-matrix interface, resulting in the change of dimensional and physical properties of the bulk materials, especially under high temperature [8]. Nevertheless, we did not observe cracks in oil-treated bamboo scrimbers after 4-hour immersion at $100^{\circ} \mathrm{C}$ (Figure $6(\mathrm{C})$ ) and $150^{\circ} \mathrm{C}$ (Figure 6(D)). This result showed that water, compared with oil, was a suitable medium to test the dimensional and physical properties of bamboo scrimbers within a short time. As shown in Figure 7, no surface cracks were observed in treated bamboo scrimbers after 4 hours of air drying at $63^{\circ} \mathrm{C}$ (Figure $7(\mathrm{E})$ ) and $100^{\circ} \mathrm{C}$ (Figure $7(\mathrm{~F})$ ). Cracks appeared in bamboo scrimbers after the treatment temperature was raised to $150^{\circ} \mathrm{C}$ (Figure $7(\mathrm{G})$ ) for $0.5 \mathrm{~h}$. However, the cracks disappeared with the drying time. The same results could be observed in Figure $7(\mathrm{H})$. After the 4-hour infrared drying treatment, the size and numbers of cracks decreased and even some cracks disappeared. This could be explained by the 


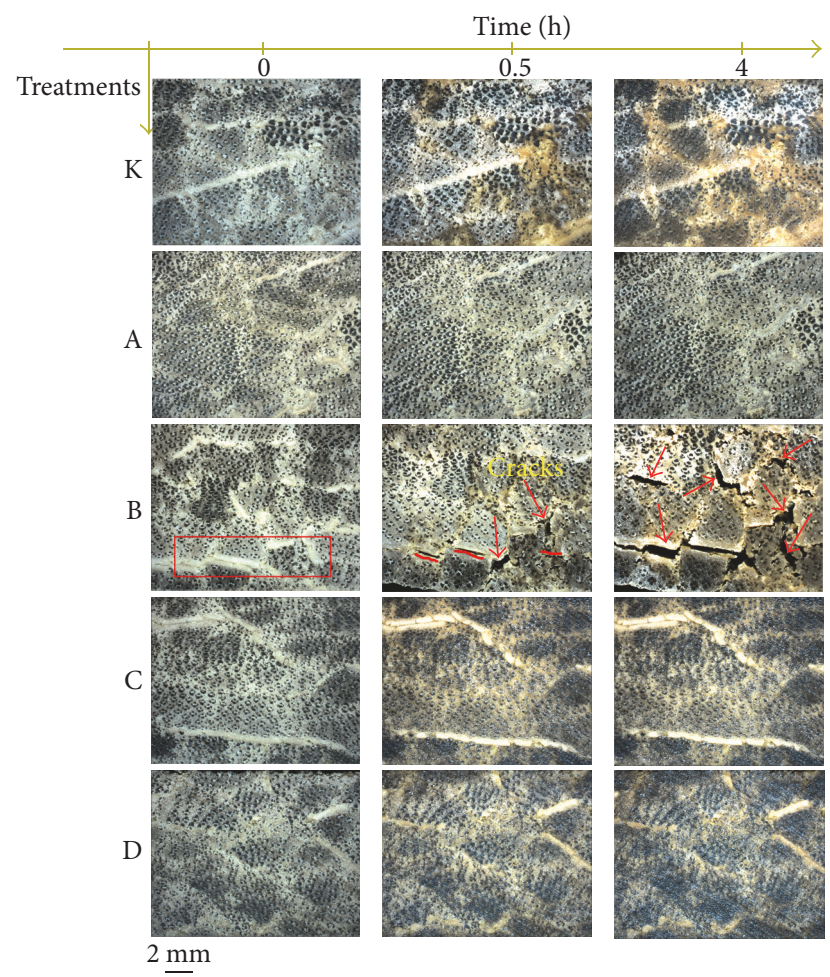

FIGURE 6: Representative optical images of the cross-section of bamboo scrimbers during the 4-hour treatments of K, A, B, C, and D.

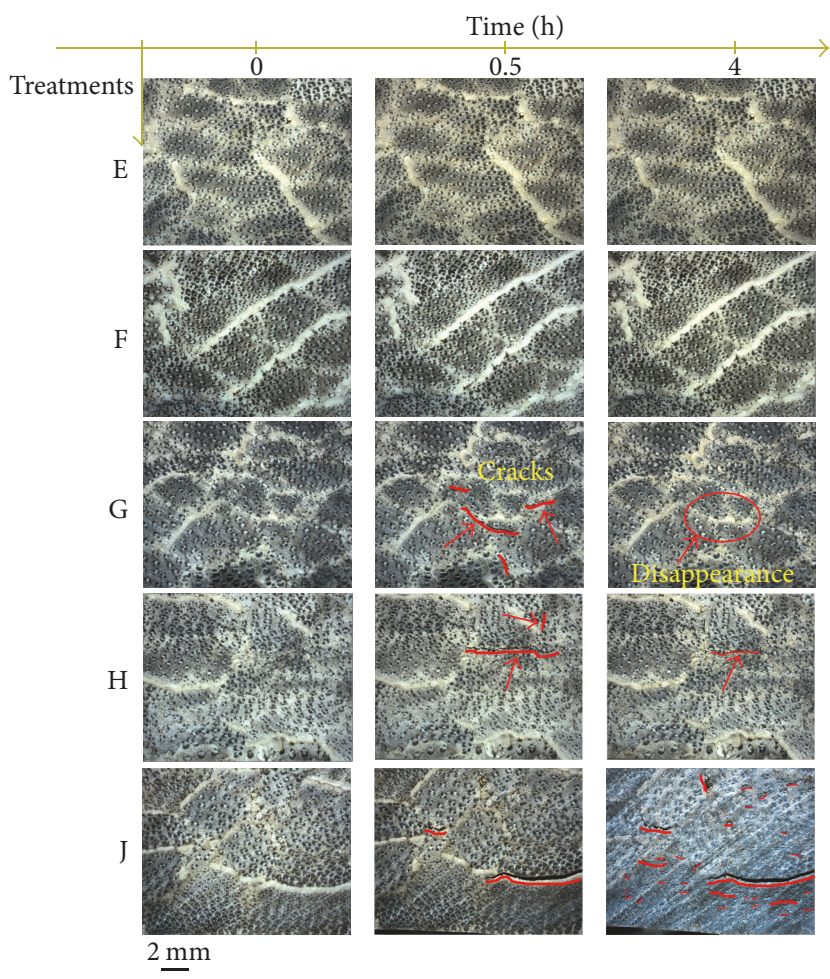

FIGURE 7: Representative optical images of the cross-section of bamboo scrimbers during the 4-hour treatments of E, F, G, H, and J. gradual decrease of moisture in bamboo scrimbers during the 4-hour drying. The rate of moisture loss in and out of bamboo scrimbers was not uniform. The surface layers of the bamboo scrimbers first lost moisture, and then the internal layers of bamboo timber lost moisture [21]. This can quickly lead to the cracking of bamboo scrimbers after 0.5 hours of air drying at $150^{\circ} \mathrm{C}$ or infrared drying at $100^{\circ} \mathrm{C}$. With the increase of treatment time, the moisture in bamboo scrimbers gradually decreased and finally maintained equilibrium. Therefore, the cracks in bamboo scrimbers gradually disappeared. However, the size and number of cracks in bamboo scrimbers increased with the treatment time with infrared drying at $150^{\circ} \mathrm{C}$ (Figure $7(\mathrm{~J})$ ). From Figure 2(J), we can conclude that the bamboo scrimber was excessively charred after 4 hours of infrared drying at $150^{\circ} \mathrm{C}$, which gave it a darker shade. Darkening occurring from the treatment was due to colored compounds being developed during the chemical changes of lignin, hemicelluloses, and the extractives [15]. Finally, the size and number of cracks in bamboo scrimbers increased with the infrared drying time.

Based on the above experimental results, we found that the water medium sensitively changed the dimension and physical properties of bamboo scrimbers compared to the others. We can use the water medium to quickly test the cracking behavior and qualified rate of bamboo products. From the results shown in Figure 7, the high temperature of the air drying or infrared drying treatment can cause cracks and color change in the bamboo scrimbers. We need to choose an appropriate drying temperature to treat the products according to one's demand. The color of the oiltreated bamboo scrimbers was found to be homogenous without any resin patches. Oil as a treating medium provides an oxygen-free environment and uniform heat can be transferred from the oil to bamboo scrimbers compared to air drying or infrared drying; thus, the color was more uniform [22]. In addition, the physical property and cracking behavior of bamboo scrimbers were relatively stable during the oil treatment process. More interestingly, we found that the bamboo scrimbers became more water repellent after the oil treatment. Further work is currently in progress in our laboratory to optimize the process parameters of oil treatments.

\section{Conclusion}

The present work focused on the influence of media treatments such as water, oil, air drying, and infrared drying on color changes, dimensional stability, and cracking behavior of bamboo scrimbers. The experimental results showed that the bamboo scrimber specimens became darker after all treatment conditions, especially the oil treatments and infrared drying. However, the color of the oil-treated bamboo scrimbers was found to be more homogenous than the others. The dimensional stability of bamboo scrimbers was more or less influenced by water immersion, air drying, and infrared drying, and the oil-treated specimens were relatively stable. Moreover, during the 4-hour treatments, cracks were found in the bamboo scrimbers after air drying at $150^{\circ} \mathrm{C}$ or infrared drying at $100^{\circ} \mathrm{C}$ or $150^{\circ} \mathrm{C}$. 


\section{Conflicts of Interest}

The authors declare that there are no conflicts of interest regarding the publication of this paper.

\section{Acknowledgments}

The work was financially supported by the Fundamental Research Funds for the Central Non-Profit Research Institution of CAF (CAFYBB2016ZD008; CAFYBB2017MA023), the Cooperation Project of Zhejiang Province and China Forestry Academy (2013SY06), and Key Laboratory of High Efficient Processing of Bamboo of Zhejiang Province (2014F10047).

\section{References}

[1] X. Liu, G. D. Smith, Z. Jiang et al., "Nomenclature for engineered bamboo," Bioresources, vol. 11, no. 1, pp. 1141-1161, 2016.

[2] L. Keogh, P. O'Hanlon, P. O'Reilly, and D. Taylor, "Fatigue in bamboo," International Journal of Fatigue, vol. 75, pp. 51-56, 2015.

[3] C.-H. Lee, M.-J. Chung, C.-H. Lin, and T.-H. Yang, "Effects of layered structure on the physical and mechanical properties of laminated moso bamboo (Phyllosachys edulis) flooring," Construction and Building Materials, vol. 28, no. 1, pp. 31-35, 2012.

[4] C. S. Verma and V. M. Chariar, "Development of layered laminate bamboo composite and their mechanical properties," Composites Part B: Engineering, vol. 43, no. 3, pp. 1063-1069, 2012.

[5] B. Sharma, A. Gatóo, M. Bock, and M. Ramage, "Engineered bamboo for structural applications," Construction and Building Materials, vol. 81, pp. 66-73, 2015.

[6] B. Sharma, A. Gatóo, and M. H. Ramage, "Effect of processing methods on the mechanical properties of engineered bamboo," Construction and Building Materials, vol. 83, pp. 95-101, 2015.

[7] A. Kumar, T. Vlach, L. Laiblova et al., "Engineered bamboo scrimber: Influence of density on the mechanical and water absorption properties," Construction and Building Materials, vol. 127, pp. 815-827, 2016.

[8] Y. Yu, R. Zhu, B. Wu, Y. Hu, and W. Yu, "Fabrication, material properties, and application of bamboo scrimber," Wood and Science Technology, vol. 49, no. 1, pp. 83-98, 2015.

[9] Y. Yu, X. Huang, and W. Yu, "A novel process to improve yield and mechanical performance of bamboo fiber reinforced composite via mechanical treatments," Composites Part B: Engineering, vol. 56, pp. 48-53, 2014.

[10] R. Zhu, Y. Zhang, and W. Yu, "Outdoor exposure tests of bamboo-fiber reinforced composite: evaluation of the physical and mechanical properties after two years," European Journal of Wood and Wood Products, vol. 73, no. 2, pp. 275-278, 2015.

[11] J. Xie, J. Qi, T. Hu, C. F. De Hoop, C. Y. Hse, and T. F. Shupe, "Effect of fabricated density and bamboo species on physical-mechanical properties of bamboo fiber bundle reinforced composites," Journal of Materials Science, vol. 51, no. 16, pp. 7480-7490, 2016.

[12] W. Shangguan, Y. Gong, R. Zhao, and H. Ren, "Effects of heat treatment on the properties of bamboo scrimber," Journal of Wood Science, vol. 62, no. 5, pp. 383-391, 2016.
[13] C. G. Du, J. G. Song, and Y. X. Chen, "The effect of applying methods of fire retardant on physical and mechanical properties of bamboo scrimber," Advanced Materials Research, vol. 1048, pp. 465-468, 2014.

[14] B. Esteves, A. Velez Marques, I. Domingos, and H. Pereira, "Heat-induced colour changes of pine (Pinus pinaster) and eucalypt (Eucalyptus globulus) wood," Wood and Science Technology, vol. 42, no. 5, pp. 369-384, 2008.

[15] M. K. Dubey, S. Pang, and J. Walker, "Effect of oil heating age on colour and dimensional stability of heat treated Pinus radiata," European Journal of Wood and Wood Products, vol. 69, no. 2, pp. 255-262, 2011.

[16] K. Srinivas and K. K. Pandey, "Effect of heat treatment on color changes, dimensional stability, and mechanical properties of wood," Journal of Wood Chemistry and Technology, vol. 32, no. 4, pp. 304-316, 2012.

[17] B. Esteves and H. Pereira, "Quality assessment of heat-treated wood by NIR spectroscopy," Holz als Roh - und Werkstoff, vol. 66, no. 5, pp. 323-332, 2008.

[18] S. Grelier, A. Castellan, and D. P. Kamdem, "Photoprotection of copper-amine-treated pine," Wood and Fiber Science, vol. 32, no. 2, pp. 196-202, 2000.

[19] P. Bekhta and P. Niemz, "Effect of high temperature on the change in color, dimensional stability and mechanical properties of spruce wood," Holzforschung, vol. 57, no. 5, pp. 539-546, 2003.

[20] M. Sailer, A. Rapp, and H. Leithoff, "Improved resistance of Scots pine and spruce by application of an oil-heat treatment," International Research Group Wood Pre, 2006.

[21] P. N. Peralta and A. W. C. Lee, "Unsteady-state diffusion of moisture in giant timber bamboo (Phyllostachys bambusoides Sieb. and Zucc," Wood Fiber Sci, vol. 27, pp. 421-427, 1995.

[22] M. K. Dubey, S. Pang, and J. Walker, "Changes in chemistry, color, dimensional stability and fungal resistance of Pinus radiata D. Don wood with oil heat-treatment," Holzforschung, vol. 66, no. 1, pp. 49-57, 2012. 


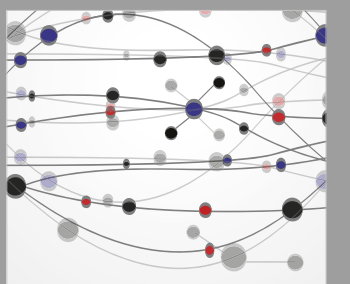

The Scientific World Journal
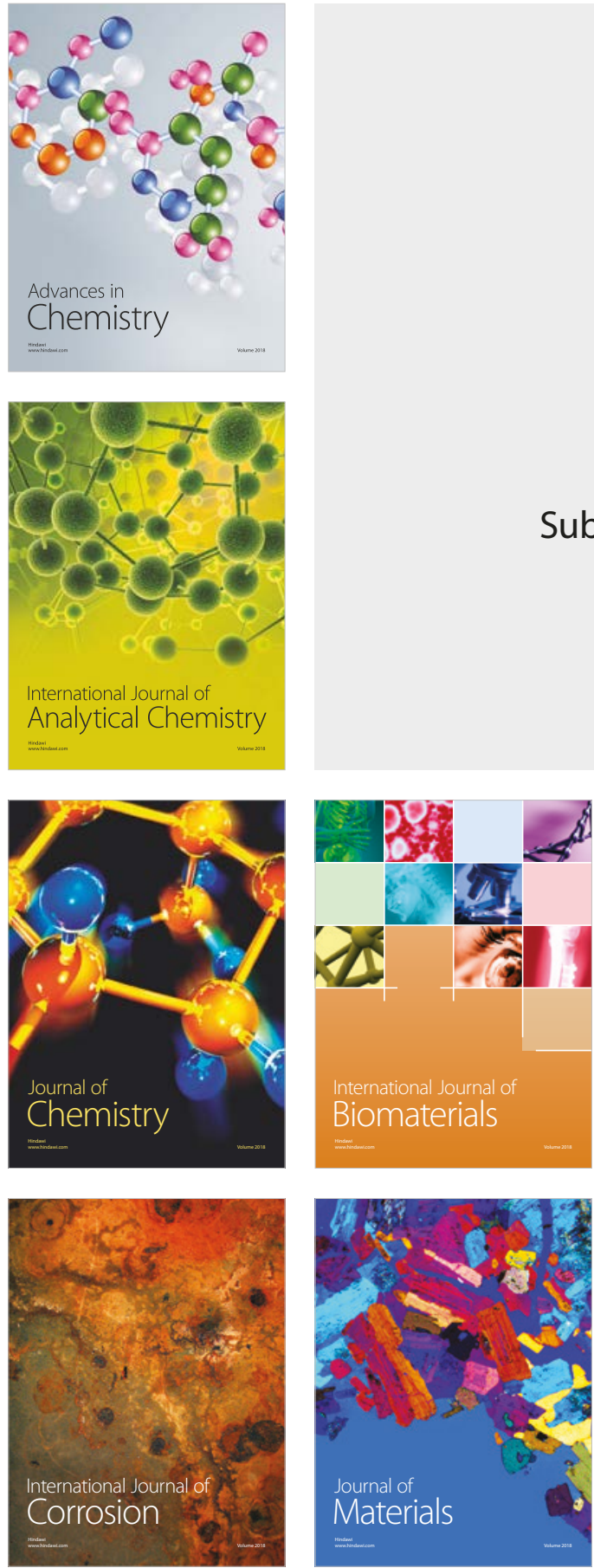

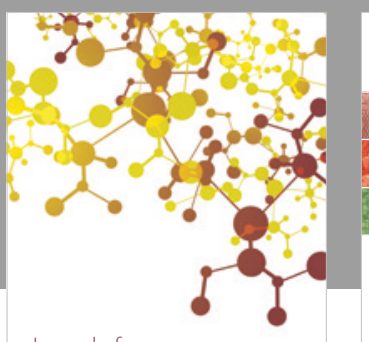

Journal of

Applied Chemistry
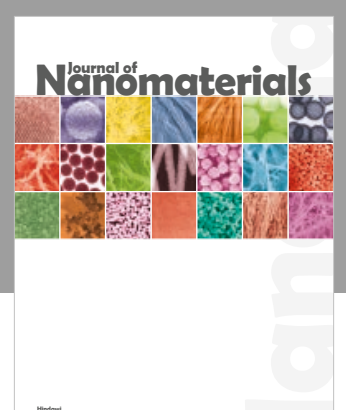

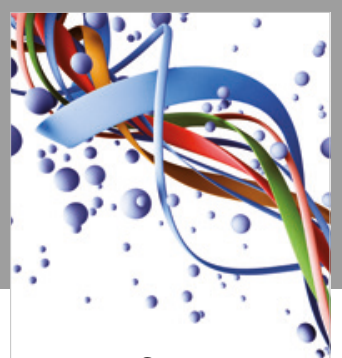

Scientifica

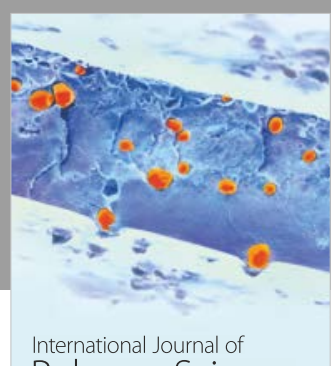

Polymer Science

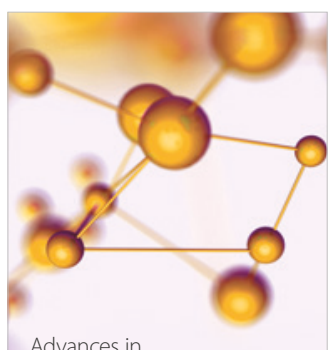

Physical Chemistry
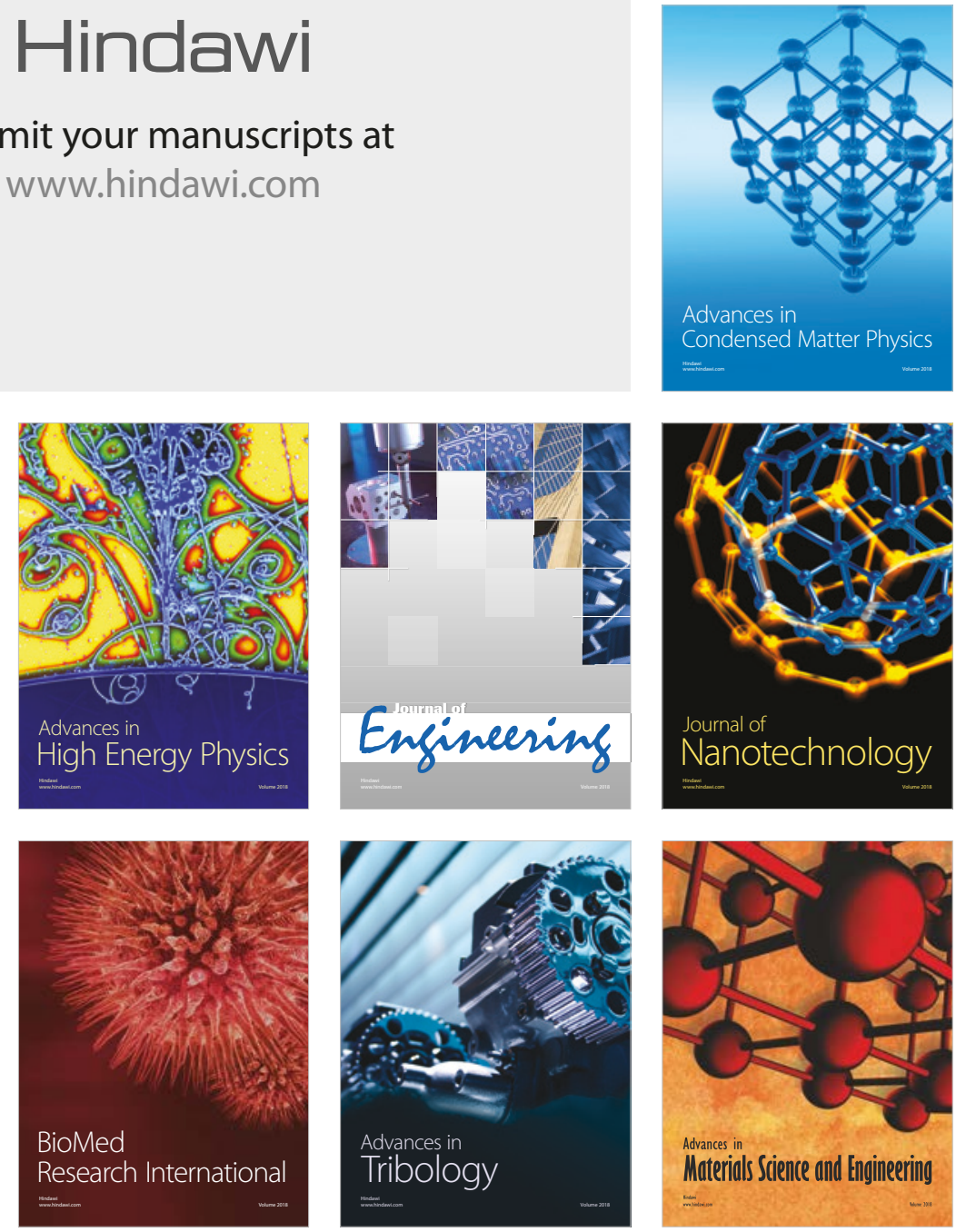